\title{
HOXB4 knockdown reverses multidrug resistance of human myelogenous leukemia K562/ADM cells by downregulating P-gp, MRP1 and BCRP expression via PI3K/Akt signaling pathway
}

\author{
HONG WANG $^{1 *}$, XIU-HONG JIA ${ }^{1 *}$, JIE-RU CHEN ${ }^{1}$, YING-JIE YI ${ }^{1}$, \\ JIAN-YONG WANG ${ }^{1}$, YOU-JIE $\mathrm{LI}^{2}$ and SHU-YANG XIE ${ }^{2}$ \\ ${ }^{1}$ Department of Pediatrics, The Affiliated Hospital of Binzhou Medical University, Binzhou, Shandong 256603; \\ ${ }^{2}$ Department of Biochemistry and Molecular Biology, Key Laboratory of Tumour Molecular Biology, \\ Binzhou Medical University, Yantai, Shandong 264003, P.R. China
}

Received August 7, 2016; Accepted October 14, 2016

DOI: 10.3892/ijo.2016.3738

\begin{abstract}
Multidrug resistance (MDR) plays a pivotal role in human chronic myelogenous leukemia (CML) chemotherapy failure. MDR is mainly associated with the overexpression of drug efflux transporters of the ATP-binding cassette (ABC) proteins. Phosphoinositide 3-kinase (PI3K)/Akt signaling cascade is involved in the MDR phenotype and is correlated with multidrug resistance 1 (MDR1)/P-glycoprotein (P-gp), multidrug resistance-associated protein 1 (MRP1) and breast cancer resistance protein (BCRP) expression in many human malignancies. Homeobox (HOX) B4, a member of the HOX gene family, has been reported to be correlated with occurrence, development, poor prognosis and drug resistance of human leukemia. In the present study, HOXB4 expression was analyzed in K562 cell line and its MDR subline K562/ADM. Compared with K562 cells, drug-resistant K562/ADM cells demonstrated evidently higher HOXB4 expression. In addition, we firstly investigated the reversal effect of HOXB4 deletion on K562/ADM cells and the underlying mechanism. The Cell Counting kit-8 (CCK-8) and flow cytometry assays showed that knockdown of HOXB4 enhanced chemosensitivity and decreased drug efflux in K562/ADM cells. Moreover, HOXB4 knockout led to downregulation of P-gp, MRP1 and BCRP expression and PI3K/Akt signaling activity, suggesting that repression of HOXB4 might be a key point to reverse MDR of K562/ADM cells.
\end{abstract}

\section{Introduction}

CML, a type of hematological malignancy, is characterized by a reciprocal chromosomal translocation $\mathrm{t}(9 ; 22)(\mathrm{q} 34 ; \mathrm{q} 11)$

Correspondence to: Professor Xiu-Hong Jia, Department of Pediatrics, The Affiliated Hospital of Binzhou Medical University, Binzhou, Shandong 256603, P.R. China

E-mail: jiaxiuhong001@163.com

Key words: HOXB4, multidrug resistance, chronic myeloid leukemia, phosphoinositide 3-kinase, P-glycoprotein, MRP1, BCRP named the Philadelphia chromosome which fuses the Abelson kinase (ABL) gene from chromosome 9 with the breakpoint cluster region (BCR) gene on chromosome $22(1,2)$. BCR-ABL with constitutive tyrosine kinase activity can phosphorylate and activate many signaling molecules leading to cell proliferation and survival (3). Clinically, tyrosine kinase inhibitors (TKIs) targeting BCR-ABL such as imatinib, nilotinib, dasatinib and ponatinib, are frontline drugs used in CML therapy $(4,5)$. Although generation of TKIs have dramatically improved the prognosis of CML, a significant portion of patients failed chemotherapy due to drug resistance $(6,7)$. TKIs resistance is a process including BCR-ABL-dependent and -independent mechanisms (8). The latter contains increase of intracellular TKIs efflux, intolerance to TKIs and the development of the phenomenon known as MDR (9). MDR is often associated with the overexpression of ABC transporters represented by MDR1/P-gp, MRP1 and BCRP, which powerfully extrude a wide variety of structurally diverse chemotherapeutics across the membranes, resulting in a decreased intracellular drug concentration and chemotherapy bioavailability $(10,11)$.

The HOX genes include a large family of homeodomaincontaining transcription factors, characterized by highly conserved DNA- and co-factor binding domains (12). In mammals, there are four such separate clusters (A-D), involving a total of $39 \mathrm{HOX}$ genes, which are key regulators of embryonic development, hematopoietic differentiation and leukemogenesis (13). We previously found that HOXA5 knockdown in U937 human leukemia cells exerted antiproliferative effects, induced apoptosis and cell cycle arrest and enhanced the cytotoxicity of cytarabine (14). Besides, silencing of HOXA10 significantly increased the cytotoxicity of ADR in K562/ADM cells; the effect of silencing HOXA10 is associated with the increased intracellular accumulation of ADR, as well as the inhibition of the expression of P-gp and MRP1 (15). HOXB4, a member of the HOX gene family, is essential in maintaining quiescent hematopoietic stem cells in steady state, adult myelopoiesis and self-renewal of the hematopoietic stem cell population $(16,17)$. It is worth noting that HOXB4 has been implicated as a cancer-related gene in many malignancies, including leukemia (18), breast (19), 
lung cancer (20), extra-hepatic cholangiocarcinoma (21) and thyroid tumours (22). The aberrant expression of HOXB4 is often related to poor prognoses of leukemia (23), mesothelioma (24) and ovarian cancer (25). Overexpression of HOXB4 is considered as a catalyst for the development of leukemia and influences the leukemia phenotype (26). Furthermore, Lehne et al (27) discovered that HOXB4 played an important role in chemoresistance of CML.

The aberrant activation of the PI3K/Akt cell transduction pathway has been found in different types of neoplasms, which contributes collectively to inhibit cell apoptosis, promote tumor proliferation, angiogenesis, invasion, metastasis and autophagy $(28,29)$. An increasing amount of preclinical data suggested that the PI3K/Akt pathway was also relevant to the MDR of different types of human malignancies, including leukemia, breast carcinoma and gastric cancer (30-32). Our previous study confirmed that the MDR K562/ADM cells demonstrated higher PI3K/Akt activity than K562 cells which was in accordance with the MDR phenotype (33). Furthermore, accumulating evidence revealed that there was a positive relationship between the expression of $\mathrm{ABC}$ proteins and the activity of PI3K/Akt signaling pathway (33-35). Nevertheless, little is known regarding the effect of the PI3K/Akt signaling on HOXB4, HOXB4-mediated leukemia MDR. Besides, the efficacy and the associated molecular mechanisms of HOXB4 suppression on the reversal of MDR, to date, have not been examined in MDR leukemia cells.

In the present study, the HOXB4 expression in K562 cell line and its MDR subline K562/ADM was analyzed. In addition, the chemosensitivity and drug efflux activity of K562/ADM cells transfected with HOXB4 short hairpin RNA (shRNA) were detected to appraise the connection between HOXB4, MDR, and the PI3K/Akt pathway, and evaluate HOXB4 as a potential molecular-target for reversing MDR of human CML K562/ADM cells.

\section{Materials and methods}

Cell lines and cell culture. Human CML K562 and its MDR counterpart K562/ADM cells were cultured as previously described (33). Human cervical carcinoma cell line HeLa was obtained from Key Laboratory of Tumour Molecular Biology of Binzhou Medical University (Binzhou, China). The cells were maintained in RPMI-1640 medium supplemented with $10 \%$ fetal bovine serum (FBS; both from HyClone Laboratories, Logan, UT, USA), at $37^{\circ} \mathrm{C}$ containing $5 \% \mathrm{CO}_{2}$.

Cell transfection. Four pairs of shRNA sequences targeting HOXB4, termed HOXB4 shRNA-1, $-2,-3$ and -4 and negative control (NC) with or without FAM-labeled were purchased from Shanghai GenePharma, Co., Ltd. (Shanghai, China). Their target sequences are as follows: HOXB4 shRNA-1, GC GAAGATGGATCCACGTTTC; HOXB4 shRNA-2, GGTAA CACACACACACTCTCC; HOXB4 shRNA-3, GCCTTGACA ACTCAGGAGTGA; HOXB4 shRNA-4, AGCAGAAGCC TCTCTCCTAGA; NC shRNA, GTTCTCCGAACGTGTCA CGT. The four shRNAs specific for HOXB4 were tested for inhibitory activity by transient transfection into HeLa cells. Briefly, cells were seeded at a density of $1 \times 10^{5} /$ well into a 6 -well plate the day before the transfection. After incubating for $24 \mathrm{~h}$, the cells were transfected with shRNAs using Lipofectamine 2000 transfection reagent (Invitrogen, Carlsbad, CA, USA) according to the manufacturer's instruction. After incubating for $6 \mathrm{~h}$, the medium was removed and replaced with the fresh one. Transfection efficiency was observed with a fluorescence microscope. RT-PCR, RT-qPCR and western blot analyses were performed to determine the inhibitory efficacy. The most effective sequence was chosen to transfect K562/ADM cells according to the manufacturer's instructions. After $48 \mathrm{~h}$ of transfection, G418 (500 ng/ml; Life Technologies, Carlsbad, CA, USA) was added to the medium. The stable positive clones were obtained after four weeks of selection.

ADM accumulation assay. Cells transfected with shRNA-3 and NC shRNA were seeded in the 6-well plate at the density of $5 \times 10^{5} /$ well, and the group without transfection was defined as a blank control. A total of $5 \mu \mathrm{M}$ ADM (Melone Pharmaceutical, Co., Ltd., Dalian, China) was applied to the wells. After incubation for $1 \mathrm{~h}$, the cells were harvested by centrifugation and washed twice with ice-cold phosphate-buffered saline (PBS). The cell-associated mean fluorescence intensity (MFI) of ADM was detected by flow cytometer using a FACSCalibur (Beckman Coulter, Brea, CA, USA) with excitation/emission wavelengths of $485 / 580 \mathrm{~nm}$.

Rhodamine-123 (Rho-123) and 5(6)-carboxyfluorescein diacetate (CFDA) accumulation assay. Rho-123 and CFDA (both from Sigma-Aldrich, St. Louis, MO, USA) were, respectively, used to evaluate the transport function of P-gp and MRP1 in K562/ADM cells by flow cytometric analysis. NC shRNA-transfected, shRNA-3-transfected and control K562/ADM cells (5x10\%/well) were seeded into 6-well plates. Subsequently, Rh-123 $(5 \mu \mathrm{M})$ or CFDA $(2 \mu \mathrm{M})$ was added, the cells were incubated for $1 \mathrm{~h}$, harvested, washed twice with cold PBS and then were measured using a flow cytometer at $488 \mathrm{~nm}$ excitation and $530 \mathrm{~nm}$ emission.

CCK-8 assay for proliferation activity. K562/ADM cells $\left(1 \times 10^{4}\right)$ with or without transfection of shRNA/well were seeded into 96-well plates and incubated with the medium containing anticancer drugs (ADM, etoposide, vindesine or cytarabine) in different concentrations for $24 \mathrm{~h}$. Each group has five parallel wells. After that, $10 \mu \mathrm{l}$ of CCK-8 (Dojindo Molecular Technologies, Inc., Shanghai, China) solution was added to every well and incubated for a further $4 \mathrm{~h}$. Then, the absorbance at $570 \mathrm{~nm}$ was measured using fluorescence. The reversal fold $(\mathrm{RF})$ values were calculated using the following formula: $\mathrm{RF}=\mathrm{IC}_{50}$ of shRNA-3 group/ $/ \mathrm{IC}_{50}$ of control group.

Reverse transcription polymerase chain reaction (RT-PCR) and reverse transcription-quantitative polymerase chain reaction $(R T-q P C R)$. Total RNA was isolated using TRIzol reagent (Invitrogen/Life Technologies) in accordance with the manufacturer's protocol and the purity of the total RNA was assessed from the ratio of A260/A280 by spectrophotometer (NanoDrop 2000; NanoDrop Technologies, Inc., Wilmington, DE, USA). A total of 1-2 $\mu \mathrm{g}$ of RNA was applied for the synthesis of the first strand cDNA. The primers (Table I) used in this experiments were designed using Primer 5 version 5.6.0 
Table I. Primers used in reverse transcription-quantitative polymerase chain reaction.

\begin{tabular}{llr}
\hline Gene & \multicolumn{1}{c}{ Primer sequence } & Product length (bp) \\
\hline HOXB4 & Forward: 5'-GCAAAGAGCCCGTCGTCT-3' & Reverse: 5'-GAAATTCCTTCTCCAGCT-3' \\
MDR1 & Forward: 5'-GGAGCCTACTTGGTGGCACATAA-3' & 138 \\
& Reverse: 5'-TGGCATAGTCAGGAGCAAATGAAC-3' & 121 \\
MRP1 & Forward: 5'-CAGCCCTTCCTGACAAGCTA-3' \\
BCRP & Reverse: 5'-GTGGCCTCATCCAACACAAG-3' \\
& Forward: 5'-GAAACCTGGTCTCAACGC-3' & 133 \\
& Reverse: 5'-AGAGTGCCCATCACAACA-3' & 189 \\
& Forward: 5'-TCCTTCCTGGGCATGGAGTC-3' & Reverse: 5'-GTAACGCAACTAAGTCATAGTC-3' \\
\hline
\end{tabular}

HOXB4, homeobox genes B4.

software and synthesized by Sangon Biotech, Co., Ltd. (Shanghai, China). The reverse transcription reaction was implemented with PrimeScript ${ }^{\mathrm{TM}}$ RT reagent kit with gDNA Eraser (Takara Bio, Shiga, Japan). The polymerase chain reaction amplification was performed on Eppendorf Mastercycler personal (Eppendorf, Co., Ltd., Shanghai, China) by using Premix Tap ${ }^{\mathrm{TM}}$ (Takara Bio). The reaction system contained diethyl pyrocarbonate, forward primer, reverse primer, Premix Tap and template cDNA. After 5 min of initial incubation at $95^{\circ} \mathrm{C}$, cDNA was amplified in 35 cycles consisting of $40 \mathrm{sec}$ denaturation at $95^{\circ} \mathrm{C}, 30 \mathrm{sec}$ annealing at $58^{\circ} \mathrm{C}$ and $40 \mathrm{sec}$ elongation at $72^{\circ} \mathrm{C}$, the PCR products were separated by $1.5 \%$ agarose gels (Takara Bio), stained with ethidium bromide for $15 \mathrm{~min}$. The images were captured by Tanon Gis systerm and $\beta$-actin served as an internal standard for quality control and quantification of target genes. RT-qPCR was performed on an ABI PRISM 7500 real-time PCR system (Applied Biosystems, Foster City, CA, USA) by using SYBR-Green reaction kit (Takara Bio). The reaction system of PCR was: SYBR-Green reagent, forward primer, reverse primer, template cDNA and nuclease-free distilled water. The PCR conditions were $95^{\circ} \mathrm{C}$, $30 \mathrm{sec}$, followed by 50 cycles of $95^{\circ} \mathrm{C}, 5 \mathrm{sec}, 60^{\circ} \mathrm{C}, 30 \mathrm{sec}$ and $\beta$-actin was used as an internal control.

Western blotting assay. Cells cultured in the 6-well plates were washed with PBS twice and proper amount of lysis buffer (Beyotime Institute of Biotechnology, Shanghai, China) was added later on. Protein concentrations were determined by Bicinchoninic acid protein assay kit (Beyotime Institute of Biotechnology). Protein samples were separated with 10 or $6 \%$ SDS-PAGE gel (Beyotime Institute of Biotechnology) and transferred onto polyvinylidine difluoride (PVDF) membranes (EMD Millipore, Bedford, MA, USA). The membranes were blocked by $5 \%$ skimmed dry milk in TBS containing $0.2 \%$ Tween-20 at room temperature for $2 \mathrm{~h}$ and incubated overnight at $4^{\circ} \mathrm{C}$ with primary antibodies. Monoclonal rabbit anti-human $\mathrm{P}$-gp and polyclonal rabbit anti-human antibodies against Akt, p-Akt (Ser473) and p-Akt (Thr308) (both 1:1,000) were purchased from Cell Signaling Technology, Inc. (Danvers, MA, USA). Rabbit polyclonal antibodies including HOXB4,
MRP1, BCRP, NF- $\mathrm{B}$ (both 1:500) and $\beta$-actin (1:3,000) were products of Beijing Biosynthesis Biotechnology, Co., Ltd. (Beijing, China). After three washes in TBS/Tween buffer, the membranes were incubated in horesradish peroxidaselabeled goat anti-rabbit immunoglobulin G (1:5,000; Beijing Zhongshan Golden Bridge Biotechnology, Co., Ltd., Beijing, China) for $2 \mathrm{~h}$ at $37^{\circ} \mathrm{C}$. Detection was performed using the FluorChem FC2 gel imaging system (Alpha Innotech Corp., San Leandro, CA, USA). Each band density was quantified using ImageJ image processing program and normalized by $\beta$-actin for their respective lanes.

Data analysis. Statistical analyses were done using SPSS 16.0 software (IBM SPSS, Armonk, NY, USA). Independent twosample t-test was used to compare the differences between the two groups. One-way analysis of variance (ANOVA) with the multiple comparison test was used to analyze the differences between three or more groups. Data were expressed as the means \pm SD. Statistical significance was accepted at $\mathrm{P}<0.05$.

\section{Results}

HOXB4 expression in $K 562$ and K562/ADM cells. We determined the expression of HOXB4 in the sensitive K562 cells and the resistant K562/ADM cells. Results demonstrated that the K562 and K562/ADM cells exhibited high expression of HOXB4. The K562/ADM cells with higher expression of P-gp, MRP1 and BCRP also showed higher HOXB4 expression than the K562 cells (Fig. 1). Hence, the K562/ADM cell line was chosen for the next HOXB4 interference.

Transfection with shRNAs to silence HOXB4. To evaluate the effects of shRNAs on knockdown of HOXB4, we transfected four HOXB4 shRNAs and one NC shRNA into HeLa cells, with cells untreated serving as a control. We tested transfection efficiency through transfection of the cells with FAM-labeled shRNAs. Green fluorescence can only be detected in the cells that were successfully transfected with FAM-labeled shRNAs (Fig. 2). Cells (84.96 $\pm 1.32 \%$ ) had green fluorescence, indicating high efficiency of the transfection. As shown in 

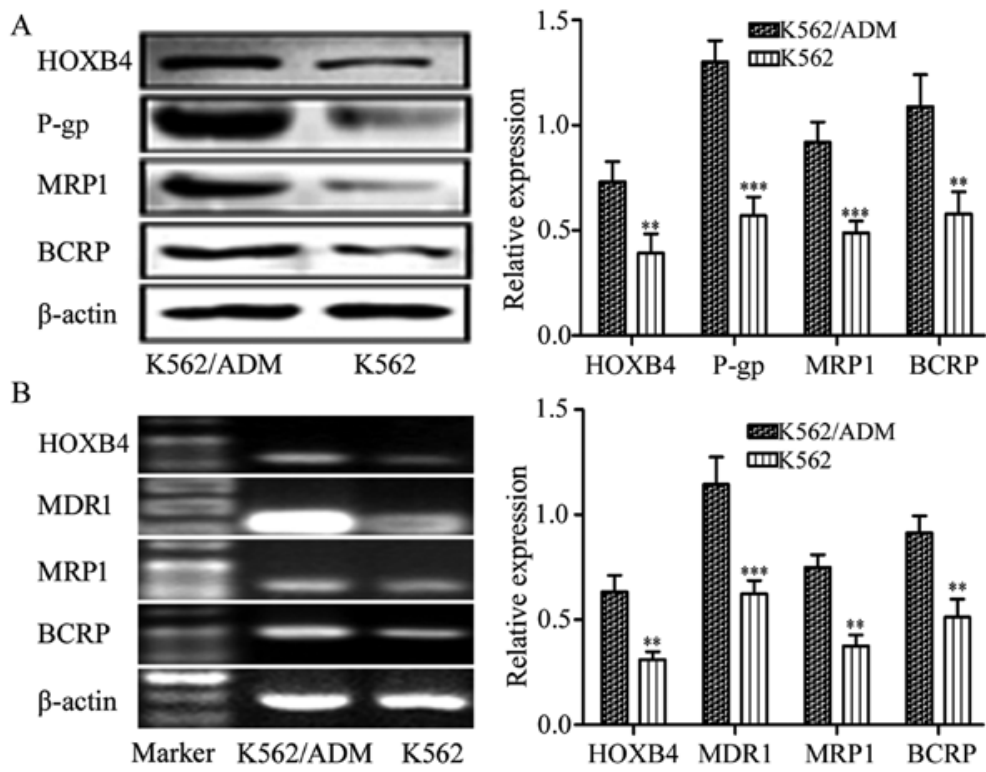

Figure 1. HOXB4 expression in K562 and K562/ADM cells. (A and B) Left panel, the HOXB4, P-gp/MDR1, MRP1 and BCRP expression in K562 or K562/ADM cells were analyzed by western blotting and RT-PCR; right panel; densitometric analysis for the detected protein expression (20 bp marker for HOXB4, MRP1, BCRP and $\beta$-actin; 100 bp marker for MDR1). All graphs show means \pm SD of three independent experiments. ${ }^{* *} \mathrm{P}<0.01$ and ${ }^{* * * *} \mathrm{P}<0.001$ compared with the K562 cells.
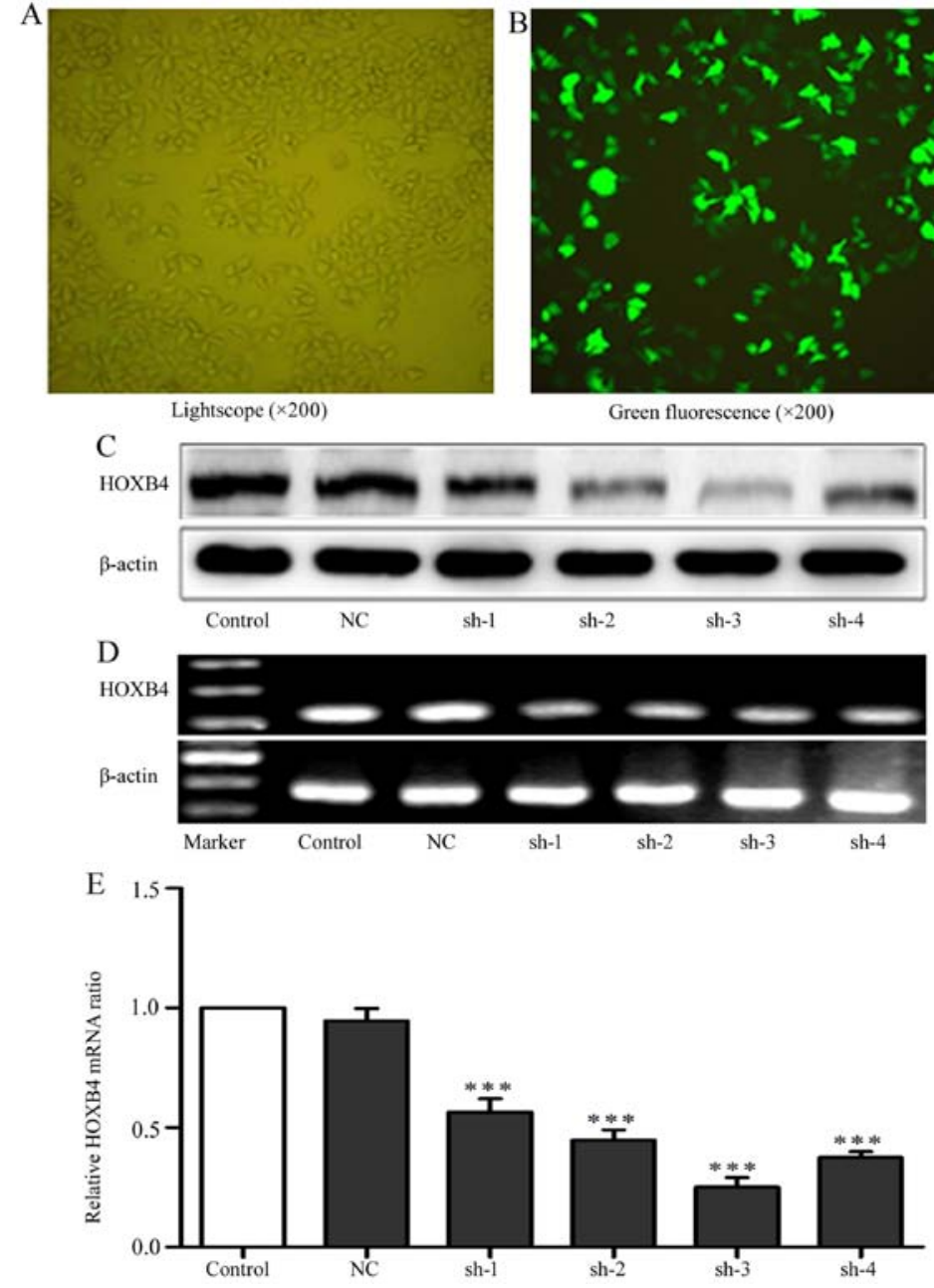

Figure 2. Transfection efficiency of HOXB4 shRNAs. (A and B) The phase contrast or the fluorescence photomicrograph in the same vision field of the HeLa cells transfected with shRNAs against HOXB4 at 24 h. (C-E) The HOXB4 protein expression of HeLa cells without transfection or transfected with NC-shRNA, shRNA-1, -2, -3 and -4 were detected by western blotting, RT-PCR and RT-qPCR (100 bp marker for HOXB4 and $\beta$-actin). Control refers to the cells without transfection. NC, sh-1, sh-2, sh-3 and sh-4 refer to cells tranfected with NC shRNA, HOXB4 shRNA-1, $-2,-3$ and -4 respectively. Data are expressed as means $\pm \mathrm{SD}$ values of triplicate experiments. ${ }^{* * *} \mathrm{P}<0.001$ vs. the control group. 
Table II. Effect of silencing HOXB4 on the sensitivity of K562/ADM cells toward four chemotherapeutic drugs by CCK-8 assay (means \pm SD of triplicate experiments).

\begin{tabular}{lccr}
\hline Treatment & Control $\left(\mathrm{IC}_{50} \mu \mathrm{g} / \mathrm{ml}\right)$ & $\mathrm{NC}$ shRNA $\left(\mathrm{IC}_{50} \mu \mathrm{g} / \mathrm{ml}\right)$ & shRNA-3 $\left(\mathrm{IC}_{50} \mu \mathrm{g} / \mathrm{ml}\right)$ \\
\hline Adriamycin & $40.47 \pm 3.85$ & $39.46 \pm 0.83$ & $7.72 \pm 0.56^{\mathrm{a}}$ \\
Etoposide & $47.24 \pm 1.41$ & $46.13 \pm 2.64$ & $22.93 \pm 1.04^{\mathrm{a}}$ \\
Vindesine & $26.55 \pm 2.95$ & $25.36 \pm 2.35$ & $14.65 \pm 0.79^{\mathrm{b}}$ \\
Cytarabine & $27.79 \pm 0.82$ & $27.27 \pm 1.78$ & $14.21 \pm 0.50^{\mathrm{a}}$ \\
\hline
\end{tabular}

${ }^{\mathrm{a}} \mathrm{P}<0.001,{ }^{\mathrm{b}} \mathrm{P}<0.01$, vs. Control and NC shRNA groups.

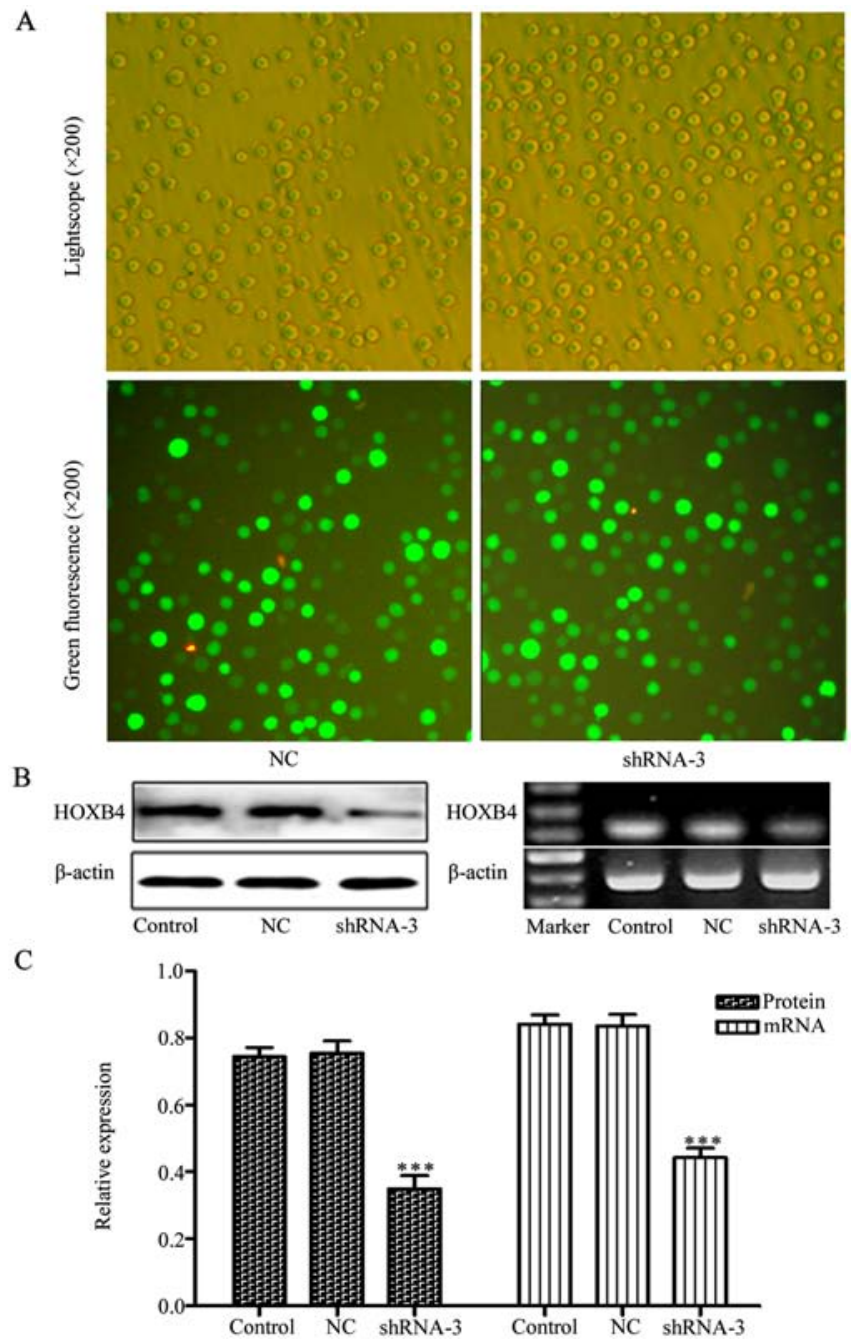

Figure 3. Transfection of HOXB4 shRNA-3 in K562/ADM cells. (A) The transfection efficiency was detected by fluorescence microscopy; the average transfection efficiency of each group was $>90 \%$ after one month of G418 selection, representative images are shown. (B and C) The protein and mRNA expression levels of HOXB4 were significantly repressed by the transfection of shRNA-3 in K562/ADM cells (100 bp marker for HOXB4 and $20 \mathrm{bp}$ marker for $\beta$-actin). NC and shRNA-3 represent K562/ADM cells tranfected with NC shRNA and HOXB4 shRNA-3. All data are presented as means $\pm \mathrm{SD} .{ }^{* * *} \mathrm{P}<0.001$ vs. the control group.

Fig. 2C-E, shRNA-3 was the most efficient plasmid to downregulate HOXB4 expression and was used to silence HOXB4 gene in K562/ADM cells. We next transfected shRNA-3 and NC shRNA into K562/ADM cells. After four weeks of G418
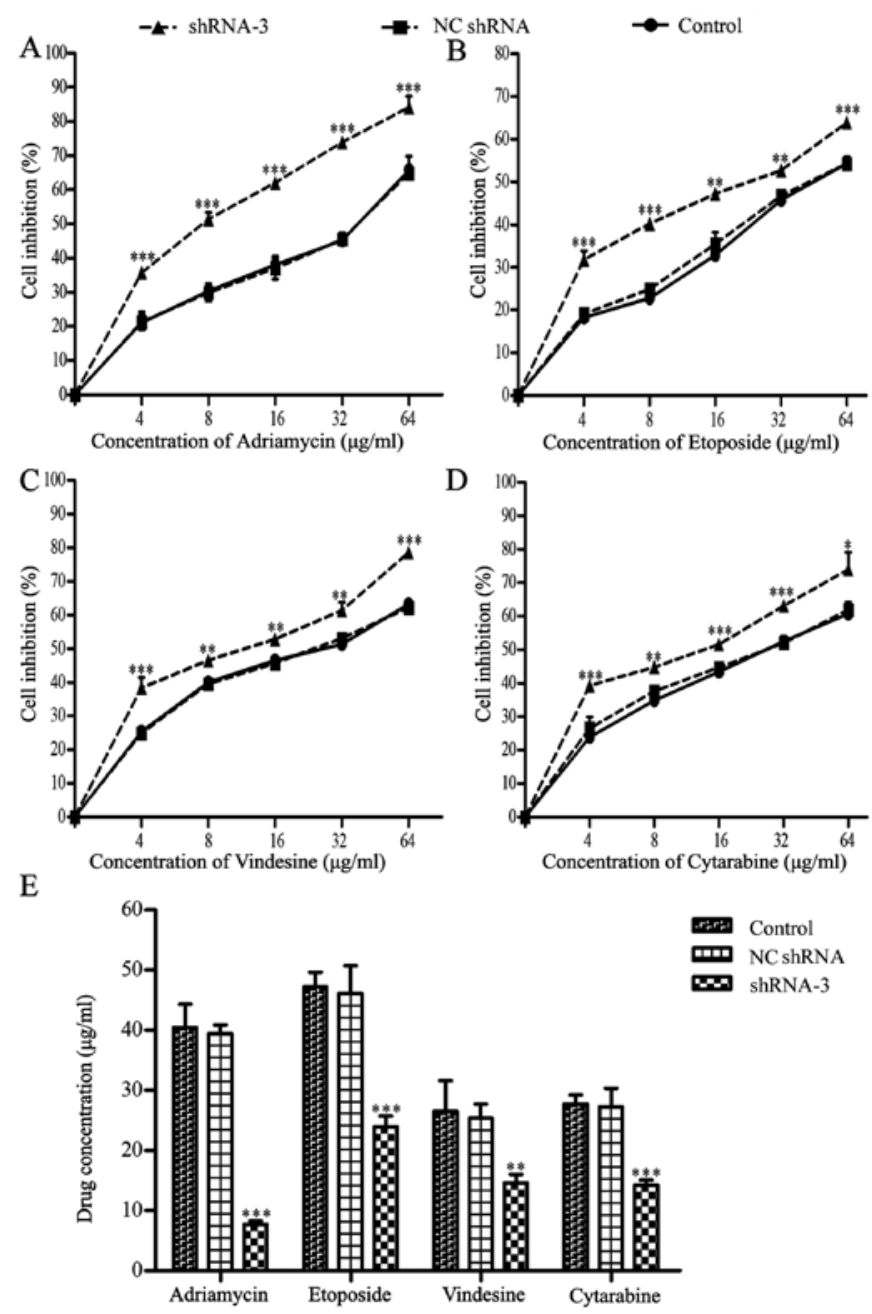

Figure 4. HOXB4 silencing restores the sensitivity of K562/ADM cells to anticancer drugs. K562/ADM cells transfected with shRNA-3 or NC-shRNA were treated with four anticancer drugs in different concentrations, and the cells without transfection were set as the control. The viability of cells was analyzed with CCK-8 assays in triplicate. (A-D) The dose-response curve of cells exposed to adriamycin, etoposide, vindesine or cytarabine, respectively. (E) Each column showed $\mathrm{IC}_{50}$ values (mean $\pm \mathrm{SD}$; $=3$ ) of corresponding groups in (A-D) calculated by the probit analysis. ${ }^{*} \mathrm{P}<0.05,{ }^{* *} \mathrm{P}<0.01$ and ${ }_{* * * *} \mathrm{P}<0.001$ compared to the control group.

selection, we successfully obtained the stable positive clones (Fig. 3). Western blotting and RT-PCR analyses revealed that HOXB4 expression decreased obviously in shRNA-3 group both in protein level and mRNA level compared with control group. While HOXB4 expression in the NC shRNA group was 

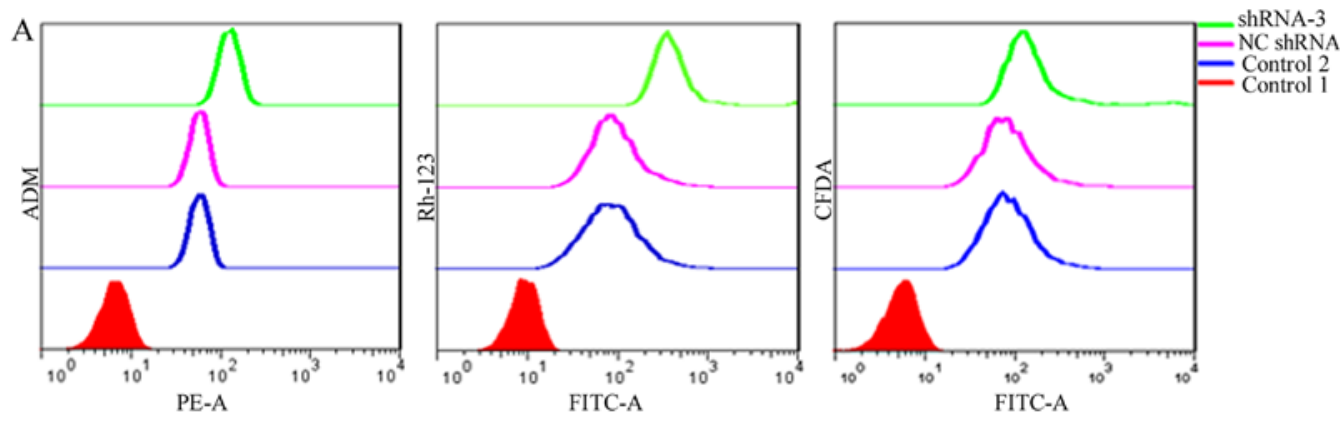

B

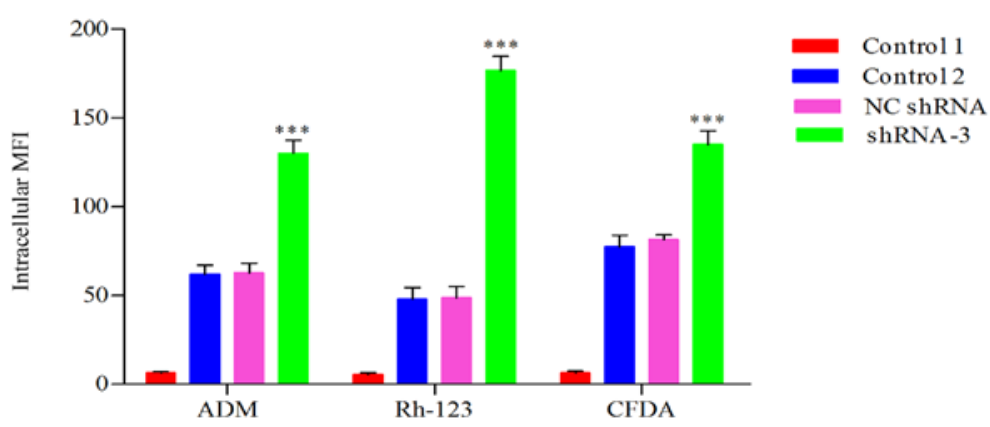

Figure 5. Increased intracellular accumulation of ADM, Rh-123 and CFDA in K562/ADM cells due to HOXB4 inhibition. (A) The intracellular accumulation of ADM, Rh-123 and CFDA were increased significantly in cells transfected with shRNA-3. (B) Enhanced uptake of ADM, Rh-123 and CFDA presented in terms of MFI values. Control 1 refers to cells without any treatment; control 2 refers to cells treated with ADM, Rh-123 and CFDA; NC shRNA and shRNA-3 refer to cells transfected with NC-shRNA or HOXB4 shRNA-3 were treated with ADM, Rh-123 and CFDA, respectively. All data are presented as means \pm SD. ${ }^{* * *} \mathrm{P}<0.001$ compared to control 2.
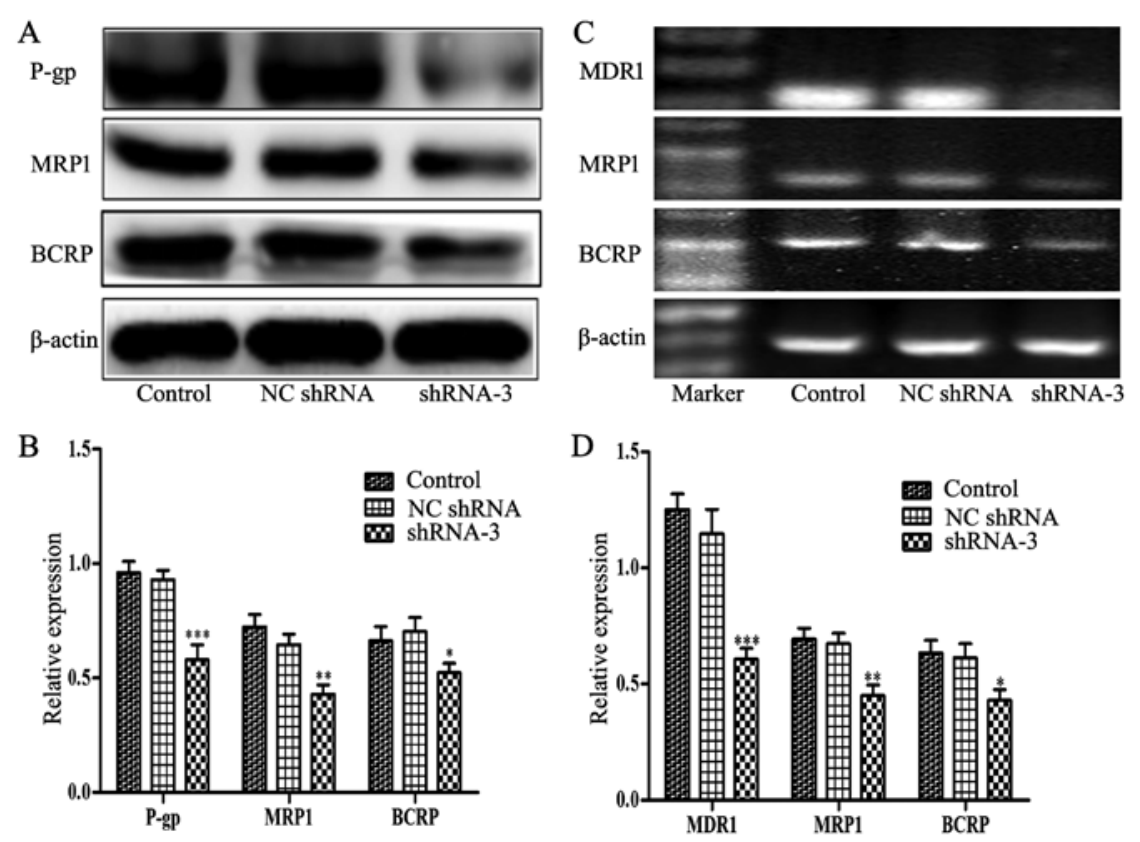

Figure 6. Knockdown of HOXB4 decreases P-gp, MRP1 and BCRP expression in K562/ADM cells. (A and C) The protein and mRNA expression of P-gp, MRP1, BCRP in K562/ADM cells transfected with shRNA-3, NC-shRNA or without transfection were detected by western blotting and RT-PCR (100 bp marker for MDR1, 20 bp marker for MRP1, BCRP and $\beta$-actin). (B and D) Densitometric analysis of protein and mRNA expression. Results are expressed as the mean \pm SD of triplicate experiments. Compared with the control group, ${ }^{*} \mathrm{P}<0.05,{ }^{* * *} \mathrm{P}<0.01$ and ${ }^{* * * *} \mathrm{P}<0.001$.

not significantly different from the control group (Fig. 3B and $\mathrm{C} ; \mathrm{P}<0.001)$.

Restoration of drug sensitivity in K562/ADM cells owing to HOXB4 knockout. As shown in Fig. 4, the dose-response curves explained that the cell sensitivity in shRNA-3 group was obviously enhanced, while the differences between the
NC shRNA group and the control group were not clear enough to distinguish (Fig. 4A-D). As seen in Fig. 4E and Table II, the $\mathrm{IC}_{50}$ values of the four drugs decreased to different extent in shRNA-3 group, especially for ADM.

Enhancement of intracellular accumulation of ADM for HOXB4 deletion. We previously confirmed that the intra- 


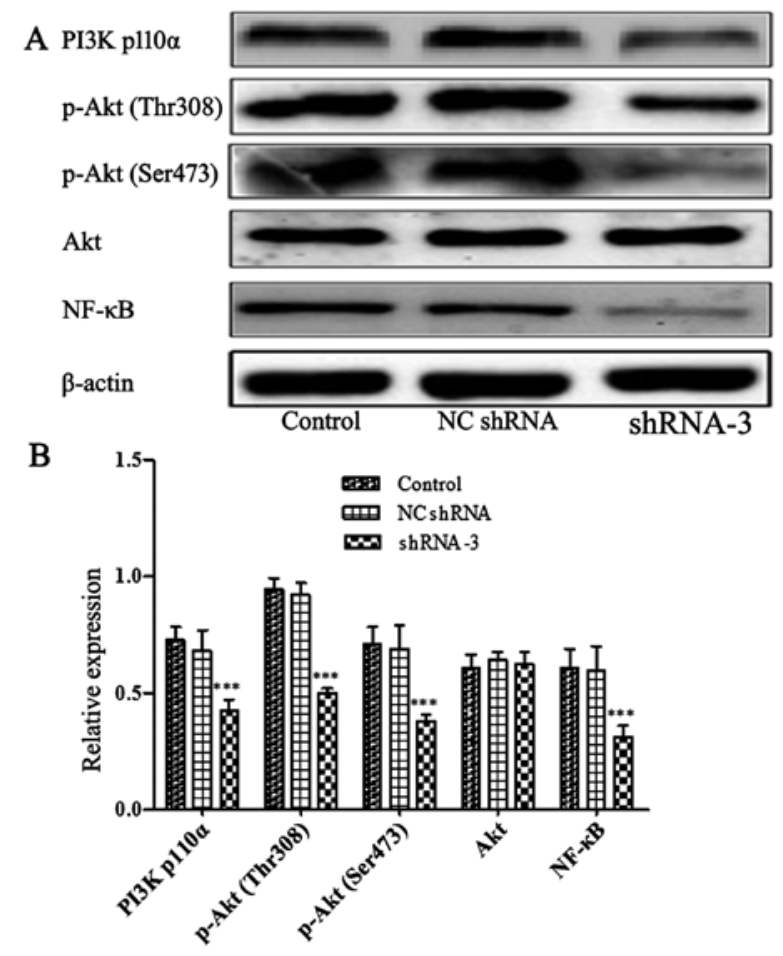

Figure 7. HOXB4 deletion blocks the activity of PI3K/Akt signaling pathway. (A) The main molecules of PI3K/Akt pathway were repressed at protein levels with shRNA-3 transfection in K562/ADM cells. (B) Densitometric analysis of protein expression. All results are expressed as the mean $\pm \mathrm{SD}$ of triplicate experiments. ${ }^{* * *} \mathrm{P}<0.001$ vs. control group.

cellular accumulation of ADM decreased significantly in K562/ADM cells compared to the parental K562 cells (33). We determined that HOXB4 deletion increased the intracellular accumulation of ADM in K562/ADM cells. As shown in Fig. 5, the accumulation value of intracellular ADM in shRNA-3 group was elevated 1.93-fold that of NC-shRNA group and 2.07-fold that of control group, respectively $(\mathrm{P}<0.001)$.

Inhibition of $P$-gp and MRPl-mediated transport due to HOXB4 suppression. The impact of HOXB4 repression on the function of P-gp and MRP1 as efflux pump in K562/ ADM cells was examined by flow cytometry. The fluorescent retention in HOXB4-silenced K562/ADM cells obviously exceeded that of NC shRNA and control groups (Fig. 5). Fig. 5 showed that the accumulation value of intracellular Rh-123 in shRNA-3 group was elevated 3.59-fold compared to NC shRNA group and 3.67-fold the control group ( $\mathrm{P}<0.001)$; the CFDA fluorescence in shRNA-3 group was enhanced 1.66-fold compared to NC shRNA group and 1.74-fold that of the control $(\mathrm{P}<0.001)$. It indicated that the efflux functions mediated by P-gp and MRP1 were significantly inhibited after HOXB4 suppression.

Decreased expression of $P$-gp, MRPI, BCRP and blockage of PI3K/Akt signaling by HOXB4 knockdown. P-gp, MRP1 and BCRP are ABC transporters overexpressed in many drug-resistant tumor cells which contributed to the development of MDR. Therefore, we assessed whether HOXB4 could influence the expression of P-gp, MRP1 and BCRP. Notably, western blotting and RT-PCR analyses (Fig. 6) illustrated that lower expression levels of P-gp, MRP1 and BCRP were detected in shRNA-3-transfected cells compared to control cells. Furthermore, Fig. 7 showed that the levels of p110 $\alpha$ (the catalytic subunit of PI3K), phosphorylation Akt at Ser473, Thr308 and NF- $\mathrm{B}$ were obviously reduced in shRNA-3 group. However, there was no change in the total amount of Akt protein. The data indicated that HOXB4 may be implicated in key steps of MDR development in CML.

\section{Discussion}

Clinically the development of MDR remains one of the major obstacles to effective tumor chemotherapy, and finding strategies to overcome it is the hotspot of current cancer research. Up to now, progress has been gained in revealing the mechanisms of MDR including alteration of the cell environment, inhibition of apoptosis and drug efflux mediated by ABC transporters (36). Our present results implied the role HOXB4 played in mediating MDR development in human CML and its possible mechanisms. Besides, we evaluated the efficacy of HOXB4 as a target therapy for overcoming drug resistance of K562/ADM cells for the first time. Aberrant expression of HOXB4 has been detected in a broad range of human carcinomas including leukemia. A previous study proposed that resistant K562/VCR expressing high level MDR genes $\mathrm{ABCB} 1$ and $\mathrm{ABCB} 4$ displayed significantly increased HOXB4 expression than parental P-gp negative K562 cells (27). In this study, upregulation of HOXB4 was also observed in MDR K562/ADM cells with higher P-gp, MRP1 and BCRP expression, which indicated that HOXB4 was also involved in the development of MDR in K562/ADM cells. To explore the connection between HOXB4 and MDR, drug-resistant K562/ADM cells were transfected with shRNA against HOXB4 and measured in chemosensitivity. Results demonstrated that HOXB4 knockout cells obviously restored the chemosensitivity to anticancer drugs such as ADM, etoposide, vindesine or cytarabine. Moreover, ADM, Rh-123 and CFDA efflux assays were exploited to assess the inhibition of $\mathrm{ABC}$ transports by HOXB4 repression and to estimate the interaction between HOXB4 and these proteins. Consistent with the speculation, efflux pump activities mediated by these ABC transporters were inhibited evidently after deletion of HOXB4. Then molecular mechanism study illustrated that there was an interrelationship between HOXB4 and these ABC proteins: HOXB4 expression in resistant K562/ADM cell line was higher than its parent K562 cell line; notably, P-gp, MRP1 and BCRP expressions at both the transcription and translation levels were inhibited significantly in response to suppression of HOXB4. It might be a reasonable explanation of chemosensitivity restoration after HOXB4 suppression when associated with the downregulation of P-gp, MRP1 and BCRP. In summary, HOXB4 repression partially reversed MDR of K562/ADM cells by inhibiting the cellular efflux function and downregulating the expression level of P-gp, MRP1 and BCRP, thus, elevating intracellular chemotherapeutic accumulation.

The PI3K/Akt pathway is excessively activated in a wide variety of hematologic malignancies including CML, diffuse large B-cell lymphoma, acute myeloid leukemia, T-cell acute lymphoblastic leukemia and chronic lymphoblastic 
leukemia; it is considered to be responsible for tumorigenesis by simultaneously promoting proliferation and inhibiting apoptosis (37). The PI3K/Akt pathway controls the expression and function of many proteins that are necessary for leukemia cell MDR and has become a promising target for systemic therapy (36-38). Morishita et al (39) proposed that Akt phosphorylation can induce chemotherapeutic resistance in B-pre-acute lymphoblastic leukemia. Moreover, several lines of evidence implicated that the maintaining of MDR in cancer cells by PI3K/Akt signaling pathway was mostly correlated with P-gp, MRP1 and BCRP $(35,36,40)$. Our previous study demonstrated that the resistant cell line K562/ADM presented higher PI3K/Akt activity than the sensitive one, which was in accordance with the MDR phenotype; we also found a parallel relationship between the activity of Akt and expression of P-gp and MRP1 $(33,34)$. In the present study, to understand why HOXB4 inhibition decreased the expression level of P-gp, MRP1 and BCRP, we evaluated more precisely the PI3K/Akt signaling expression alteration in the case of HOXB4 deletion. Decreased expression of HOXB4 showed obviously lower protein expression of the main signal molecules of PI3K/Akt signaling in K562/ADM cells. These results indicated that HOXB4-modulated MDR in CML K562/ADM cell line was, at least in part, PI3K/Akt-dependent.

In conclusion, HOXB4 was expressed at a higher level in the K562/ADM cells, and knockdown of HOXB4 enhanced the sensitivity of the K562/ADM cells to cytotoxic killing by the therapeutic drugs, such as ADM, etoposide, vindesine or cytarabine, as a result of the increased intracellular accumulation of chemotherapeutics. The inhibition of drug efflux induced by HOXB4 deletion was associated with downregulation of P-gp, MRP-1 and BCRP proteins. Moreover, the relationships between HOXB4 and PI3K/Akt signaling pathway were further elucidated, with a conclusion that interference of HOXB4 was able to reverse MDR in K562/ADM cells via repression of PI3K/Akt activity. While the initial findings are promising, more comprehensive and detailed studies still need to be conducted, such as animal models in vivo or more precise PI3K/Akt pathway assays. Above all, we suggest that knockdown of HOXB4 is a novel and potent therapeutic target that could be used for reversing MDR in human CML K562/ ADM cells.

\section{Acknowledgements}

The present study was supported by the Natural Science Foundation of Shandong Province (nos. ZR2014HL032 and ZR2014HQ079) and the Projects of Medical and Health Technology Development Program in Shandong Province (no. 2014WS0183).

\section{References}

1. Cao R, Wang Y and Huang N: Discovery of 2-acylaminothiophene-3-carboxamides as multitarget inhibitors for BCR-ABL kinase and microtubules. J Chem Inf Model 55: 2435-2442, 2015

2. Mathisen MS, Kantarjian HM, Cortes J and Jabbour E: Mutant BCR-ABL clones in chronic myeloid leukemia. Haematologica 96: 347-349, 2011

3. Yandim MK, Ceylan C, Elmas E and Baran Y: A molecular and biophysical comparison of macromolecular changes in imatinibsensitive and imatinib-resistant K562 cells exposed to ponatinib. Tumour Biol 37: 2365-2378, 2016.
4. O'Hare T, Shakespeare WC, Zhu X, Eide CA, Rivera VM, Wang F, Adrian LT, Zhou T, Huang WS, Xu Q, et al: AP24534, a pan-BCR-ABL inhibitor for chronic myeloid leukemia, potently inhibits the T315I mutant and overcomes mutation-based resistance. Cancer Cell 16: 401-412, 2009.

5. Sweet K and Pinilla-Ibarz J: Early switch in tyrosine kinase inhibitor therapy for patients with chronic myeloid leukemia: An emerging clinical question. Crit Rev Oncol Hematol 103: 99-108, 2016.

6. Hochhaus A, Ernst T, Eigendorff E and La Rosée P: Causes of resistance and treatment choices of second- and third-line treatment in chronic myelogenous leukemia patients. Ann Hematol 94 (Suppl 2): S133-S140, 2015.

7. La Rosée P, Corbin AS, Stoffregen EP, Deininger MW and Druker BJ: Activity of the Bcr-Abl kinase inhibitor PD180970 against clinically relevant $\mathrm{Bcr}-\mathrm{Abl}$ isoforms that cause resistance to imatinib mesylate (Gleevec, STI571). Cancer Res 62: 7149-7153, 2002

8. Corrêa S, Binato R, Du Rocher B, Castelo-Branco MT, Pizzatti L and Abdelhay E: Wnt/ $\beta$-catenin pathway regulates ABCB1 transcription in chronic myeloid leukemia. BMC Cancer 12: 303, 2012.

9. Gottesman MM: Mechanisms of cancer drug resistance. Annu Rev Med 53: 615-627, 2002.

10. Ambudkar SV, Kimchi-Sarfaty C, Sauna ZE and Gottesman MM: P-glycoprotein: From genomics to mechanism. Oncogene 22: 7468-7485, 2003.

11. Kalle AM, Sachchidanand S and Pallu R: Bcr-Abl-independent mechanism of resistance to imatinib in K562 cells: Induction of cyclooxygenase-2 (COX-2) by histone deacetylases (HDACs). Leuk Res 34: 1132-1138, 2010.

12. Hoegg S and Meyer A: Hox clusters as models for vertebrate genome evolution. Trends Genet 21: 421-424, 2005.

13. Cillo C, Cantile M, Faiella A and Boncinelli E: Homeobox genes in normal and malignant cells. J Cell Physiol 188: 161-169, 2001.

14. Li N, Jia X, Wang J, Li Y and Xie S: Knockdown of homeobox A5 by small hairpin RNA inhibits proliferation and enhances cytarabine chemosensitivity of acute myeloid leukemia cells. Mol Med Rep 12: 6861-6866, 2015.

15. Yi YJ, Jia XH, Wang JY, Li YJ, Wang H and Xie SY: Knockdown of HOXA10 reverses the multidrug resistance of human chronic mylogenous leukemia K562/ADM cells by downregulating P-gp and MRP-1. Int J Mol Med 37: 1405-1411, 2016.

16. Kavalerchik E, Goff D and Jamieson CH: Chronic myeloid leukemia stem cells. J Clin Oncol 26: 2911-2915, 2008.

17. Thorén LA, Liuba K, Bryder D, Nygren JM, Jensen CT, Qian H, Antonchuk J and Jacobsen SE: Kit regulates maintenance of quiescent hematopoietic stem cells. J Immunol 180: 2045-2053, 2008.

18. Zhang XB, Beard BC, Trobridge GD, Wood BL, Sale GE, Sud R, Humphries RK and Kiem HP: High incidence of leukemia in large animals after stem cell gene therapy with a HOXB4expressing retroviral vector. J Clin Invest 118: 1502-1510, 2008.

19. Bodey B, Bodey B Jr, Siegel SE and Kaiser HE: Immunocytochemical detection of the homeobox B3, B4, and C6 gene products in breast carcinomas. Anticancer Res 20 (5A): 3281-3286, 2000.

20. Bodey B, Bodey B Jr, Gröger AM, Siegel SE and Kaiser HE: Immunocytochemical detection of homeobox B3, B4, and C6 gene product expression in lung carcinomas. Anticancer Res 20: 2711-2716, 2000.

21. Hwang SH, Kim KU, Kim JE, Kim HH, Lee MK, Lee CH, Lee SY, Oh T and An S: Detection of HOXA9 gene methylation in tumor tissues and induced sputum samples from primary lung cancer patients. Clin Chem Lab Med 49: 699-704, 2011.

22. Rodríguez-Rodero S, Fernández AF, Fernández-Morera JL, Castro-Santos P, Bayon GF, Ferrero C, Urdinguio RG, GonzalezMarquez R, Suarez C, Fernández-Vega I, et al: DNA methylation signatures identify biologically distinct thyroid cancer subtypes. J Clin Endocrinol Metab 98: 2811-2821, 2013.

23. Jamieson CH, Ailles LE, Dylla SJ, Muijtjens M, Jones C, Zehnder JL, Gotlib J, Li K, Manz MG, Keating A, et al: Granulocyte-macrophage progenitors as candidate leukemic stem cells in blast-crisis CML. N Engl J Med 351: 657-667, 2004.

24. Morgan R, Simpson G, Gray S, Gillett C, Tabi Z, Spicer J, Harrington KJ and Pandha HS: HOX transcription factors are potential targets and markers in malignant mesothelioma. BMC Cancer 16: 85, 2016.

25. Kelly Z, Moller-Levet C, McGrath S, Butler-Manuel S, Kavitha Madhuri T, Kierzek AM, Pandha H, Morgan R and Michael A: The prognostic significance of specific HOX gene expression patterns in ovarian cancer. Int J Cancer 139: 1608-1617, 2016. 
26. Gordon-Keylock SA, Jackson M, Huang C, Samuel K, Axton RA, Oostendorp RA, Taylor H, Wilson J and Forrester LM: Induction of hematopoietic differentiation of mouse embryonic stem cells by an AGM-derived stromal cell line is not further enhanced by overexpression of HOXB4. Stem Cells Dev 19: 1687-1698, 2010.

27. Lehne G, Grasmo-Wendler UH, Berner JM, Meza-Zepeda LA Adamsen BL, Flack A, Reiner A, Clausen OP, Hovig E and Myklebost O: Upregulation of stem cell genes in multidrug resistant K562 leukemia cells. Leuk Res 33: 1379-1385, 2009.

28. Sheng Z, Ma L, Sun JE, Zhu LJ and Green MR: BCR-ABL suppresses autophagy through ATF5-mediated regulation of mTOR transcription. Blood 118: 2840-2848, 2011.

29. Xie X, Tang B, Zhou J, Gao Q and Zhang P: Inhibition of the PI3K/Akt pathway increases the chemosensitivity of gastric cancer to vincristine. Oncol Rep 30: 773-782, 2013.

30. Cheng L, Luo S, Jin C, Ma H, Zhou H and Jia L: FUT family mediates the multidrug resistance of human hepatocellular carcinoma via the PI3K/Akt signaling pathway. Cell Death Dis 4: e923, 2013.

31. Mao Z, Zhou J, Luan J, Sheng W, Shen X and Dong X: Tamoxifen reduces $\mathrm{P}$-gp-mediated multidrug resistance via inhibiting the $\mathrm{PI} 3 \mathrm{~K} / \mathrm{Akt}$ signaling pathway in ER-negative human gastric cancer cells. Biomed Pharmacother 68: 179-183, 2014.

32. Tazzari PL, Cappellini A, Ricci F, Evangelisti C, Papa V, Grafone T, Martinelli G, Conte R, Cocco L, McCubrey JA, et al: Multidrug resistance-associated protein 1 expression is under the control of the phosphoinositide 3 kinase/Akt signal transduction network in human acute myelogenous leukemia blasts. Leukemia 21: 427-438, 2007.

33. Wang H, Jia XH, Chen JR, Wang JY and Li YJ: Osthole shows the potential to overcome P-glycoprotein-mediated multidrug resistance in human myelogenous leukemia K562/ADM cells by inhibiting the PI3K/Akt signaling pathway. Oncol Rep 35 3659-3668, 2016.
34. Chen JR, Jia XH, Wang H, Yi YJ, Wang JY and Li YJ: Timosaponin A-III reverses multi-drug resistance in human chronic myelogenous leukemia K562/ADM cells via downregulation of MDR1 and MRP1 expression by inhibiting PI3K/Akt signaling pathway. Int J Oncol 48: 2063-2070, 2016.

35. Huang FF, Wu DS, Zhang L, Yu YH, Yuan XY, Li WJ, Chen XP, Zhao XL, Chen FP and Zeng H: Inactivation of PTEN increases ABCG2 expression and the side population through the PI3K/Akt pathway in adult acute leukemia. Cancer Lett 336: 96-105, 2013.

36. Ma H, Cheng L, Hao K, Li Y, Song X, Zhou H and Jia L: Reversal effect of ST6GAL 1 on multidrug resistance in human leukemia by regulating the PI3K/Akt pathway and the expression of P-gp and MRP1. PLoS One 9: e85113, 2014.

37. Zhang X, Dong W, Zhou H, Li H, Wang N, Miao X and Jia L: $\alpha-2,8$-sialyltransferase is involved in the development of multidrug resistance via PI3K/Akt pathway in human chronic myeloid leukemia. IUBMB Life 67: 77-87, 2015.

38. Cheng Z, Yang N, Liang W, Yan X, Li L and Pan L: Effect of phosphatase and tensin homology deleted on chromosome 10 (PTEN) gene transfection on reversal of multidrug resistance in K562/ADM cells. Leuk Lymphoma 53: 1383-1389, 2012.

39. Morishita N, Tsukahara H, Chayama K, Ishida T, Washio K, Miyamura T, Yamashita N, Oda M and Morishima T: Activation of Akt is associated with poor prognosis and chemotherapeutic resistance in pediatric B-precursor acute lymphoblastic leukemia. Pediatr Blood Cancer 59: 83-89, 2012.

40. Jung KA, Choi BH and Kwak MK: The c-MET/PI3K signaling is associated with cancer resistance to doxorubicin and photodynamic therapy by elevating BCRP/ABCG2 expression. Mol Pharmacol 87: 465-476, 2015 